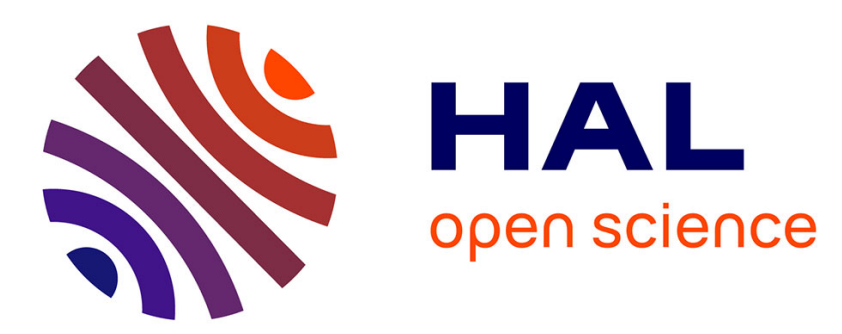

\title{
La course aux belles affaires : professionnalisme et performance du travail de la police judiciaire
}

François Dedieu

\section{To cite this version:}

François Dedieu. La course aux belles affaires: professionnalisme et performance du travail de la police judiciaire. Sociologie de l'action organisée: nouvelles études de cas, Editions De Boeck, 2011, Ouvertures sociologiques, 9782804162436 . hal-02808670

\section{HAL Id: hal-02808670 \\ https: / hal.inrae.fr/hal-02808670}

Submitted on 6 Jun 2020

HAL is a multi-disciplinary open access archive for the deposit and dissemination of scientific research documents, whether they are published or not. The documents may come from teaching and research institutions in France or abroad, or from public or private research centers.
L'archive ouverte pluridisciplinaire HAL, est destinée au dépôt et à la diffusion de documents scientifiques de niveau recherche, publiés ou non, émanant des établissements d'enseignement et de recherche français ou étrangers, des laboratoires publics ou privés. 


\title{
La course aux belles affaires : professionnalisme et performance du travail de la police judiciaire
}

\author{
François Dedieu*
}

Même si la sociologie des organisations défend une conception élargie de l'intérêt (Friedberg 1993), les usages et les interprétations de ce paradigme, ont trop souvent tendance à privilégier une lecture strcitement utilitariste de la rationalité de l'acteur (Lecas et Jobert 1980). A travers l'analyse du cas d'une bureaucratie de police judiciaire, une Direction Départementale de la Sécurité Publique (DDSP) de la banlieue parisienne, nous voudrions montrer combien les intérêts professionnels pèsent dans la regulation mixte des systèmes d'action. Non seulement les éléments constitutifs d'une profession (le mandat et la licence notamment ; Dubard, Tripier 1998) participent aux stratégies rationnelles et à la régulation des systèmes d'actions concrêts. Nous montrerons comment ces éléments relevant d'une sociologie des professions, participent à entretenir la motivation des policiers et, consécutivement, à la performance de cette unité de police judiciaire. Il s’agira de montrer, plus précisémment, que la mobilisation professionnelle au sein des unités de Police Judiciaire s'explique non pas seulement par la congruence existante entre le mandat institutionnel et les missions de ces unités - la lutte contre le crime -(Brodeur 1984), mais également par une course aux belles affaires qui permet de faire converger les intérêts intrinsèques à la profession policière avec les objectifs de résultats de cette unité. Plus

Chargé de recherche à l’INRA, unité Inra Sens et Institut Francielien Recherche Innovation (IFRIS), chercheur associé au Centre de Sociologie des Organisations. 
généralement, alors que la sociologie des organisations a eu tendance a mettre en exergue les effets de la bureaucratie, l'étude organisationnelle de cette unité de police judiciaire, démontrera comment bureaucratie peut créer des effets vertueux pour sa performance et pour la motivation de ses membres.

\section{La sûreté départementale : une bureaucratie professionnelle}

\subsection{L’organisation de la sûreté départementale}

La sûreté départementale, relevant de l'autorité de la Direction Départementale de Sécurité Publique (DDSP), mène des investigations dont les commissariats au niveau local et la police judiciaire au niveau national ne peuvent se charger. Elle traite des affaires d'une délinquance dite " moyenne ", qui requiert des moyens (en hommes et en matériel) trop importants pour les commissariats locaux mais qui restent, en même temps, d'une ampleur insuffisante pour justifier que la police judiciaire en ait la charge ${ }^{1}$.

Si la structure est composée de trois brigades, nous nous intéresserons, dans cet article, à deux d'entre elles ${ }^{2}$. La première est la brigade de sécurité urbaine (BSU). Elle est chargée de résoudre des affaires concernant des infractions de «voie publique » à savoir les vols avec violences, les vols à la roulotte, les destructions et les dégradations volontaires, les vols par ruse ou encore les cambriolages. La BSU travaille sur initiative c'est-à-dire qu'elle traite ses propres affaires

${ }^{1}$ Rappelons que les brigades de la police judicaire (la « $\left.\mathrm{PJ} »\right)$ ne traite qu'une délinquance de grande envergure telle que les braquages de banques en bandes organisées, les crimes de sang ou la délinquance financière.

${ }^{2}$ La troisième brigade de la structure est la brigade des mineurs. 
indépendamment des commissariats. Généralement, et sous réserve de l'autorisation du substitut du procureur, la BSU se saisit d'affaires initiées par les commissariats locaux et traitent de l'affaire de manière autonome. Les policiers de la BSU apportent également et ponctuellement un appui technique aux commissariats et à leurs services judiciaires ${ }^{3}$ lorsque ceux-ci sont engagés dans des opérations importantes ${ }^{4}$.

La seconde brigade étudiée est la brigade des stupéfiants qui a la charge de la répression des infractions liées à la législation des stupéfiants ${ }^{5}$. Classiquement, la brigade traite des affaires de cession et de revente dans le cadre de trafics établis à l'échelle départementale, voire régionale ${ }^{6}$. La brigade travaille également d'initiative et peut, comme la BSU, être amenée à apporter un soutien ponctuel aux commissariats. Mais pour les stupéfiants, les affaires provenant des saisines du parquet, qui sont principalement des Commissions Rogatoires (CR), sont proportionnellement plus importantes que pour la BSU?.

\footnotetext{
${ }^{3}$ Le GRI (groupe de recherche et d'investigation)

${ }^{4}$ Il peut s'agir par exemple d'une fonction d'appui lors de la constitution de procédures particulièrement lourdes qui engagent un nombre important de mis en cause. Dans ce cas, les commissariats sont les maîtres d'œuvre de la procédure. Du fait de cette mission formelle, et contrairement aux autres brigades de la structure, la BSU est très peu saisie par le procureur afin qu'elle rester disponible pour sa fonction d'appui « technique » ou aux commissariats du département.

${ }^{5}$ Réglementé par le code de santé publique.

${ }^{6}$ Les trafics nationaux et internationaux sont réservés à la Direction Régionale de la Police Judiciaire (DRPJ).

${ }^{7}$ Elles étaient de l'ordre de $20 \%$ du volume des affaires en 1999 pour la BSU et plus de $45 \%$ pour la brigade des stupéfiants. Source : rapport d'activité de la sûreté départementale 1999. Nuançons pourtant ici nos propos. Comme nous l'on précisé les acteurs, les chiffres concernant les saisines du parquet n'étaient pas réellement significatifs cette année là dans la mesure où la brigade a vocation à travailler un peu plus d'initiative. En 1999 et en 2000, les groupes composant la brigade avaient été profondément renouvelée en hommes, ce qui l'avait conduit à accepter plus de saisines du parquet afin d'assurer un volume d'activité durant cette période. Nous
} 
Chacune des deux brigades est composée de plusieurs groupes de six policiers environ qui travaillent sur des affaires la plupart du temps indépendamment des uns des autres. Au sein de la sûreté départementale, la structure hiérarchique se présente de la manière suivante :

- Le commissaire principal est le responsable de la structure. Il a la charge de la gestion du budget ainsi que celle du personnel. Dans la pratique, le commissaire s'occupe peu des affaires internes et a se focalise surtout sur la communication externe $\mathrm{e}^{8}$

- Dans les groupes d'investigation, les chefs de groupes et leurs adjoints, qui ont respectivement le grade de capitaine et de lieutenant de police, ont la responsabilité des missions de recherche et d'investigations. Ils dirigent des policiers qui sont soit officiers de police judiciaire (OPJ) ou soit gardien de la paix. Les chefs de brigades sont quant à eux commandants de police et assurent la gestion quotidienne des groupes d'investigation. Ils contrôlent l'exécution des investigations dont ils ont la responsabilité directe. Ils assument, d'autre part, des tâches de gestion de ressources humaines telles que le décompte des heures supplémentaires, les congés ou encore les récupérations.

\subsection{Le recrutement par cooptation}

Le profil des policiers de la sûreté départementale est très varié : on trouve des officiers de police judiciaire (OPJ) mais aussi des brigadiers et des gardiens de la paix issus des

reviendrons sur les raisons de ce renouvellement très important de la brigade en hommes dans la partie II.

${ }^{8}$ Il représente par exemple le service lors des réunions de concertation entre services de police de la circonscription ainsi que lors d'opérations de partenariats plus divers comme dans le cadre des plans locaux de sécurité, ou auprès des mairies par exemple. 
commissariats mais aussi des Brigades anti-criminalité (BAC) ou encore des Compagnies d'Intervention (CI) de la circonscription.

A la sûreté départementale (SD), le recrutement des policiers s'effectue en grande partie par la cooptation. Le procédé est le suivant : au cours des opérations d'investigation menées conjointement avec les commissariats, les policiers de la $\mathrm{SD}$ repèrent et testent des recrues potentielles. Dans ces situations de travail commun, les policiers de la SD évaluent les aptitudes de leurs collègues selon trois critères. Premièrement, ils doivent faire preuve d'initiative, qualité indispensable dans le travail d'investigation. Deuxièmement, le postulant potentiel doit faire la démonstration de sa capacité à travailler collectivement ${ }^{9}$. Le troisième et le plus important critère est la motivation du policier à intégrer la sûreté départementale. Mais ce critère n'est pas le plus compliqué à évaluer puisque le nombre de volontaires souhaitant intégrer la structure est très important. On peut avancer deux raisons à cela : tout d'abord, pour les policiers issus des commissariats, intégrer la sûreté départementale représente une opportunité d'ascension professionnelle puisqu'ils se verront l'opportunité de préparer le concours d'officiers de Police judiciaires (OPJ). Ensuite, et surtout, pour ces policiers issus des commissariats ou des BAC, y voient l'opportunité d'effectuer un travail de police judiciaire qui est associé au travail « noble » de la police par opposition au traitement des petites infractions et aux tâches de maintien de la paix qui composent la vie des commissariats. La réputation de la SD à résoudre des affaires d'envergure rend le service particulièrement attractif vis-à-vis des policiers des circonscriptions.

${ }^{9}$ Cela implique que le candidat ait certaines capacités relationnelles : se montrer sympathique et avoir sens de l'humour et de la dérision sont les capacités qui sont particulièrement recherchées. 
Ce système de recrutement n'est pas explicité en tant que tel et la plupart du temps, les policiers qui sont l'objet de cette évaluation n'en sont pas conscients. Lorsqu'un poste se libère, les brigades recommandent à leur groupe et à leur hiérarchie, un candidat avec lequel ils ont eu l'occasion de travailler et qu'ils jugent compétent. Le postulant est, ensuite, soumis à l'appréciation du groupe de travail dans son ensemble qui dispose d'un réel pouvoir de sanction : il est soumis à une période d'essai qui est généralement de trois semaines. A l'issue de cette période, le groupe émet avis au chef de service. S'il est négatif, et en fonction des motifs avancés, le candidat pourra être soit dirigé vers un autre groupe où il effectuera un nouvel essai ${ }^{10}$, ou soit sera réintégré dans son organisation d'origine ${ }^{11}$.

${ }^{10}$ Il sera alors nécessaire d'intervertir certains policiers entre les groupes concernés, nous avons pu observer ce type de cas au cours de nos observations.

${ }^{11}$ Les données recueillies nous ne nous ont pas permis de relever des cas d'échec patents dans la cooptation. On peut cependant imaginer que certains nouveaux recrutés ne se révèlent pas aussi efficaces. Sans nier leurs existences, trois éléments nous permettent de faire l'hypothèse, qu'il faudrait vérifier, que les échecs ont un impact minime dans la structure. Tout d'abord, et comme nous venons de le préciser, la période d'essai imposée aux candidats permet de mieux juger des capacités du candidat et par conséquent de limiter les erreurs de jugement éventuelles. Ensuite, et comme cela nous a été précisé dans les entretiens, c’est sur la partie procédurale du travail d'investigation que les nouveaux intégrés peuvent connaître le plus de difficulté. Autrement dit, c'est sur cet aspect que les risques d'échec du recrutement sont les plus grands. Or, comme nous le verrons dans la partie suivante, si le policier éprouve des difficultés trop importantes sur le travail procédural, il sera affecté à des tâches essentiellement opérationnelles (planques, arrestations, gardes 
Seul le recrutement des officiers placés à la tête des groupes échappe à ce système de cooptation. Dans ces cas, c'est la haute hiérarchie de la structure (commissaire et commissaire principal de la DDSP) qui recrute, seule, l'OPJ chef du groupe.

\section{La sureté départementale, un service efficace}

\subsection{Une évaluation sur la réputation}

La sûreté départementale est considérée comme un service d'élite par la hiérarchie de la Direction Départementale de la Sécurité Publique (DDSP) et par les magistrats du parquet. Il est évalué par la hiérarchie de la DDSP et par les magistrats sur leur réputation à résoudre de belles affaires qui se définissent de manière générique comme des affaires d'envergure, qui sortent de l'ordinaire et qui vont marquer l'esprit des acteurs.

«On n'arrive pas bien à la (l'évaluation du service) mesurer au sens où vous l'entendez, moi je vois qu' il y a la façon dont les affaires sont traitées, la façon dont ça sort, comment c'est ressenti sur le terrain, comment c'est ressenti par les magistrats, c'est plutôt par ces aspects-là /.../ Je pense que c'est une unité qui a déjà fait un travail tout à fait remarquable et je dois dire que toutes les affaires sur lesquelles ont les a branchés se sont traduites par de vrais succès, des enquêtes suffisamment en profondeur et étayées pour pouvoir mettre un véritable coup de balai a posteriori donc moi c'est à ça que je

à vue, interrogatoires). Enfin, les risques d'erreurs sont limités, selon nous, par le volume important du nombre de postulants potentiels. En effet, en ayant un choix de postulants élargi, les policiers de la sureté départementale sont plus à même à repérer de « bons » candidats. 
mesure l'efficacité » Directeur Départemental de la Sécurité Publique

Pour comprendre pourquoi la structure est jugée avant tout sur une réputation, il nous faut expliquer, au préalable, les raisons pour lesquelles les taux d'élucidation sont peu significatifs dans cette structure, contrairement aux commissariats. Comme toute structure de la DDSP, la sûreté départementale comptabilise son taux d'élucidation qui est le résultat d'un ratio entre les faits constatés ${ }^{12}$ et les faits élucidés ${ }^{13}$. Mais contrairement aux autres, la SD ne reçoit pas de plainte venant du public. Elle se saisit, la plupart du temps, d'affaires auprès des commissariats, une fois que ceux-ci ont accompli les premiers actes d'investigation. Or, le guide de méthodologie statistique de la direction nationale de la police nationale, stipule qu'un "fait constaté » ne peut être comptabilisé deux fois. Ainsi, lorsqu'un commissariat transmet une procédure à la sûreté départementale, il a déjà effectué l'exploitation statistique c'est-à-dire son enregistrement en « fait constaté ». Ceci induit donc que les brigades de la sûreté départementale élucident des faits qu'elles n'ont pas constatés ${ }^{14}$. Seules les affaires confiées par le parquet sont comptabilisées comme des «faits constatés ». Mais travaillant majoritairement d'initiative, ces affaires ne

\footnotetext{
${ }^{12}$ Plaintes déposées par le public (dans ce cas seuls les crimes et délits entrent dans ce champ statistique)

${ }^{13}$ Enquêtes ayant abouties à l'arrestation des auteurs ou à la découverte des objets dérobés

${ }^{14}$ Pour illustrer nos propos, prenons l'exemple d'un mode de calcul standard du taux d'élucidation. Mettons que pour que pour les deux infractions suivantes "Vols avec entrées par ruse en tout lieu " et "Menaces ou chantage pour extorsion de fonds» on compte respectivement pour 15 et 11 faits constatés, 7 et 3 faits élucidés. Le ratio entre faits constatés et faits élucidés donne un taux d'élucidation global de 38,46\%. Reprenons à présent le même calcul tel que le fait la sûreté départemental qui élucide des faits non comptabilisés comme "faits constatés ». Ainsi, que la sûreté départementale appréhende l'ensemble des auteurs ou seulement une partie, elle aura un taux de $100 \%$.
} 
constituent qu'une partie du travail des brigades, même si elles sont, proportionnellement plus importantes pour les stupéfiants que pour la $\mathrm{BSU}^{15}$. Par conséquent, la sûreté départementale affiche à chaque bilan, un taux d'élucidation supérieur à 100\%.

C'est donc d'abord parce que le taux d'élucidation n'est pas significatif que le service est évalué sur une réputation à résoudre des affaires "marquantes », celles que la profession dénomme les «belles » affaires. Plus généralement, la belle affaire est la manifestation concrète de ce qui constitue le cœur du métier de policier, ce qui en fait sa noblesse et son prestige. La belle affaire se définit selon des critères qualitatifs et quantitatifs ${ }^{16}$.

En premier lieu, la belle affaire est une affaire de « qualité » selon la définition qu'en donne les policiers et, plus largement, la profession policière. La qualité se définit par le résultat et la performance.

Tout d'abord, la qualité de la prise s’apprécie au regard du degré de gravité délictuelle dont on retrouve des manifestations plus ou différentes selon les spécialités. Pour les stupéfiants comme pour la BSU, il s'agit d'un trafic de drogues ou de marchandises qui a une envergure régionale, nationale voire internationale. L'affaire du « top model américain » est une des belles affaires de la brigade des stupéfiants. Le principal trafiquant était un top model célèbre qui utilisait ses réseaux parmi des célébrités du show business pour écouler des quantités importantes de drogues (herbe de cannabis et cocaïne). Ce qui fonde ici l'envergure du résultat c'est la dimension internationale de l'affaire puisque les policiers ont démantelé le réseau jusqu'en en Hollande, mais également l'originalité de l'affaire. A ce moment, ils ont eu

${ }^{16}$ Nous avons établi ces crtères à partir de cinq affaires traités par les brigades, qualifiées de « belles affaires ». 
l'occasion d'auditionner plusieurs célébrités de la chanson et du cinéma. À la BSU, la gravité de l'infraction peut être liée à la répétition de délits comme ce fût le cas dans l'affaire des «saucissonneurs »: il s'agissait d'une bande organisée qui séquestrait des personnes âgées, les attachaient les unes aux autres (d'où leur nom) en leur faisant subir des sévices corporels pour qu'elles leurs dévoilent les lieux où elles entreposaient leurs épargnes. Dans les deux spécialités, la qualité du délit s'incarne également dans l'arrestation de délinquants d'envergure, ceux que les policiers surnomment «les beaux mecs ». Le groupe 3 de la BSU bénéficie, à ce titre, d'une réputation tout à fait particulière dans le service puisqu'il a la charge des infractions et des vols "par ruse ». Ces affaires sont souvent l'occasion d'appréhender des «beaux mecs » puisque ces voleurs sont actifs et récidivistes. Les exemples sont nombreux mais citons les plus célèbres comme le " faux agent EDF » qui utilise cette fausse identité pour dérober les personnes âgées à domicile ou encore ceux surnommés les « collègues », une bande de voleur qui agissait selon un mode opératoire original: un premier voleur prétextait une excuse pour pénétrer dans l'habitation de la victime. Une fois dans l'appartement, ses complices déguisés en policiers feignaient une arrestation en flagrant délit. De cette manière, il incitait la victime à dévoiler où étaient cachés leurs biens, et de les voler plus facilement.

Ensuite, la belle affaire est l'objet d'une reconstruction a posteriori de la part des policiers investigateurs puisqu'ils y voient une performance. C'est dans ces affaires exceptionnelles que les policiers font la démonstration (plus qu'à l'ordinaire) de leurs compétences d'enquêteurs. La performance s'apprécie, de manière générale, au regard de la difficulté de la tâche qui peut se mesurer au regard de l'adversaire et de la difficulté de l'investigation. Les voleurs par ruse sont, en effet, généralement des voleurs 
professionnels. Ils se montrent particulièrement prudents en ne laissant jamais d'empreintes nettes ${ }^{17}$ et en utilisant systématiquement des véhicules non identifiables ${ }^{18}$. Du reste, les enquêtes de voisinage se révèlent, généralement, vaines puisque les voleurs exercent, entre 11 heure du matin et midi. Enfin, la majorité des victimes sont des personnes âgées qui ont très souvent les plus grandes difficultés à identifier après coups les auteurs.

Cette notion qualitative du résultat correspond à la représentation que se font les policiers se font du "vrai » travail en opposition à la répression des petits délits qui compose le travail quotidien des policiers de la rue.

En second lieu, la belle affaire revêt une notion de résultats plus tangible. La notion qualitative de la prise dans la belle affaire s'accompagne la plupart du temps d'une série de résultats chiffrés et donc mesurables: nombre d'auteurs, l'ampleur des saisies effectuées et le nombre de résolution de plaintes qu'elle a permis de résoudre.

Tout d'abord, le caractère quantitatif de la belle affaire se mesure d'abord au regard du nombre d'auteurs appréhendés et condamnés. Ainsi, dans les affaires du « dealer top model » pour les stupéfiants et dans celle des vols de voitures pour la BSU 2, ont permis d'appréhender respectivement 10 et 15 auteurs. Consécutivement, le résultat en termes d'arrestations, passe par les peines infligées aux auteurs. Les lourdes peines sont perçues comme la récompense d'un travail «bien fait »: elles relèvent des investigations efficaces qui ont permis d'accumuler des

\footnotetext{
${ }^{17}$ Le verre constitue le support idéal pour un relevé d'empreinte. Mais selon les policiers, peu d'affaires sont résolues grâce aux empreintes puisqu'il est nécessaire de relever 12 points de comparaison.

${ }^{18}$ Ils ne garent jamais leurs véhicules devant les maisons des victimes et utilisent des « doublettes », c'est à dire de fausses plaques d'immatriculation.
} 
preuves irréfutables : témoignages, des preuves matérielles ou d'autres expertises plus fines comme les tests d'ADN.

Ensuite, ce sont les saisies effectuées qui attestent de l'envergure quantitative de la «belle affaire». C'est logiquement à la brigade des stupéfiants que cet aspect est primordial. Par exemple dans l'affaire du «top model dealer ", que nous citions précédemment, les policiers saisirent, outre la cocaïne, $115 \mathrm{~kg}$ d'herbe et de cannabis. A la BSU également, les saisies peuvent marquer une affaire d'envergure. Le groupe 1 mis ainsi fin aux agissements d'un réseau de trafiquants de cartes informatiques ayant escroqué un matériel équivalent à 12 millions de francs.

"Quand on parle d'une belle affaire, il y a plusieurs éléments : c'est d'abord envoyer au trou des mecs néfastes, des méchants, c'est pour ça qu'on est flics. C'est quand t'as démantelé un trafic, coupé le trafic et saisi des éléments qui prouvent la culpabilité du mec pour qu'il aille en prison. Les peines récompensent notre travail, et quand ils (les auteurs) vont en prison c'est qu'on a assez de preuves contre eux. Le tribunal statue en fonction de la procédure pas en fonction d'une rumeur. Plus les mecs ils prennent plus t'as été bon » OPJ stup

Enfin, la résolution d'une belle affaire se mesure aux plaintes qu'elle permet de résoudre. C'est le cas notamment pour les voleurs par ruse comme le faux agent EDF qui était particulièrement actif dans le département, ce qui a permis de solutionner un grand nombre de plaintes qui étaient au départ saisies par les commissariats locaux.

Ceci n'est pas contradictoire avec ce que nous établissions plus haut concernant la faible pertinence de l'évaluation chiffrée pour mesurer l'activité de la sûreté départementale. Mais il n’en demeure pas moins que la 
résolution d'affaires d'envergure revêt une importance sur le plan comptable de la criminalité du département.

« Je ne leur ai jamais fait de remarques sur les chiffres. Non, ce sont des gens qui en veulent donc la question ne se pose pas dans les mêmes termes que dans les commissariats, là vous savez il y a de tout, $y$ ' a des gens qui en veulent et d'autres un peu moins /.../ De toute façon, cette question du taux d'élucidation, vous la retrouvez au niveau global de la circonscription. Quand la sûreté résout une affaire, on le retrouve sur les chiffres de la circonscription » Directeur départemental de sécurité publique

Terminons ce point par un bénéfice que la structure tire de la résolution des belles affaires. De part l'originalité des auteurs et de l'envergure des résultats obtenus, les belles affaires ont une résonance importante sur le plan médiatique et contribue à la publicité du service. Le commissaire de la sûreté départemental se charge d'ailleurs d'exploiter et d'entretenir la publicisation de sa structure dès qu'il le peut. Il entretien des rapports réguliers avec les journalistes de la rubrique faits d'hivers du " Parisien », journal local de grande audience $^{19}$. La presse participe à la construction de la réputation du service auprès de la hiérarchie de la DDSP, des magistrats du TGI ainsi qu'auprès des policiers des commissariats locaux, ce qui renforce un peu plus leur motivation à postuler dans la structure.

\footnotetext{
${ }^{19}$ Le journal à notamment couvert l'affaire du «top model dealer » dans ses différentes phases: l'arrestation de l'auteur, sa biographie, son procès et son jugement.
} 


\section{Les relations internes à la SD : une autonomie forte et contrôle hiérarchique distant}

\subsection{Les relations hiérarchiques : une délégation responsabilisée}

Les brigades d'investigation jouissent d'une grande autonomie : hormis la part, néanmoins importante du « travail de bureau $»^{20}$, la nature du travail d'investigation induit une faible proportion de tâches routinières ou programmées. Des évènements imprévisibles, dangereux et singuliers, pouvant revêtir un caractère d'urgence bouleversent le déroulement des enquêtes judiciaires. Dans le cas des "planques" par exemple, les policiers ne savent jamais si ces surveillances vont déboucher sur une filature qui les entraînera vers d'autres destinations ou si, au contraire, ils s’y ennuieront fermement.

L’imprévisibilité perpétuelle implique des prises de décision rapides de la part des policiers qui peuvent être lourdes conséquences pour la suite de leurs investigations. Les policiers de la brigade des stupéfiants évoquent souvent le cas suivant : lors d'une surveillance, ils aperçoivent un suspect, soupçonné d'être un important trafiquant, transporter une mallette. La décision est délicate : faut-il l'interpeller ? Transporte-t-il du produit dans sa mallette ou non ? Si oui, y en a-t-il suffisamment pour établir le trafic ? Si ce n'est pas le cas, toutes les surveillances futures seront-elles anéanties ?

Ainsi, le travail d'investigation reposant essentiellement sur l'initiative des policiers, la hiérarchie de la structure ne peut prescrire l'ensemble des tâches à accomplir et n'a pas d'autre choix que d'accorder une forte autonomie aux

${ }^{20}$ Travail qui se caractérise par l'élaboration de la procédure judiciaire mais également les gardes à vue. 
fonctionnaires. Cette autonomie est accordée à chaque strate de la hiérarchie de la SD :

$\mathrm{Au}$ niveau des groupes, les officiers responsables admettent avoir plus un rôle de coordinateur plutôt que de commandement « traditionnel » strict, tel qu'il est exercé dans les commissariats. Si le responsable du groupe a des tâches exclusives - il est l'interlocuteur unique du magistrat, il répartit les tâches pour les premiers actes d'investigation et fait la synthèse des différentes informations au gré de l'avancement de l'enquête - son mode de commandement du groupe reste très souple. Ce type de commandement est très apprécié des hommes qui le perçoivent comme une marque de confiance. Pour autant, le chef de groupe est reconnu en tant que tel puisqu'il fonde son autorité sur une expérience plus importante dans le travail d'investigation et en matière procédurale. Du reste, la petite taille des groupes atténue la distance hiérarchique formelle et qui place le chef à un niveau proche de ses hommes.

Le commissaire principal, favorise également l'autogestion des groupes. Mais cette liberté est accordée en contrepartie de résultats, c'est-à-dire en termes d'affaires de « qualité ». Si les résultats ne sont pas là, la hiérarchie se fait plus présente et peut user de son rôle de sanction formel. Nous en citons un exemple plus bas.

\subsection{Les relations dans les groupes : ajustement mutuel et préférences négociées}

L'autonomie élargie des groupes et des brigades n'en reste pas moins encadrée par des règles de fonctionnement plus ou moins explicitées. Ces règles «autonomes" concernent d'abord dans la régulation des rythmes de travail qui sont l'objet d'une négociation tacite entre les hommes et 
la hiérarchie. En effet, le système des récupérations offrant de nombreux jours de repos supplémentaires aux policiers, il est de l'intérêt du service que les policiers n'exigent pas trop fréquemment $^{21}$ leurs repos compensatoires. La négociation implicite entre l'encadrement et la brigade est alors la suivante: la hiérarchie ne contrôle pas les horaires de présence et en échange les policiers font une gestion raisonnée des récupérations et ne " compte pas leurs heures " lors des affaires exceptionnelles pouvant conduire les policiers à travailler plusieurs jours de suite. Cette négociation est reportée au niveau de la brigade : les groupes comptabilisent leurs horaires et leurs activités dans la « main courante ». Ce document est ensuite soumis au contrôle du chef de brigade qui reconnaît ne pas consulter régulièrement ${ }^{22}$. Cette gestion libre des horaires est très appréciée des policiers des groupes qui savent néanmoins ne pas abuser de ce privilège.

Ensuite, les règles autonomes concernent la division du travail. Le travail d'investigation est essentiellement collectif : selon les policiers, le travail d'investigation s'apparante à un processus itératif continu. En effet, les dossiers sont attribués d'abord individuellement afin d'effectuer les premiers actes d'enquêtes ${ }^{23}$. Mais par la suite et régulièrement, tout le groupe donne son avis sur les avancées du dossier. Par ailleurs, dans les groupes, la polyvalence des hommes est rendue obligatoire lors des surcharges de travail imprévues ${ }^{24}$. Ceci induit une relative réduction de l'écart entre les compétences des APJ et

\footnotetext{
${ }^{21}$ La règle administrative autorise un policier à prendre 30 jours consécutifs de récupérations au maximum.

${ }^{22}$ Il leur suffit, en effet, que le quota minimum de présence de $50 \%$ du personnel total soit respecté. Autrement dit, le respect du seuil garantit aux groupes la nonintervention du chef de brigade.

${ }^{23}$ Il s'agit des premiers recoupements : identités de l'entourage (famille, amis..), passé judiciaire, premières auditions

${ }^{24}$ C'est par exemple le cas pour une garde à vue de dix prisonniers. Si un membre des sept ignore comment rédiger la majorité des PV de procédure, le groupe prendra du retard.
} 
des OPJ, ces derniers étant plus volontiers spécialisés sur la rédaction d'actes procéduraux complexes comme le rapport de synthèse, concluant la procédure. À la sûreté départementale, il n'est pas rare d'observer un renversement des compétences, des jeunes OPJ moins chevronnés, laissent le soin à des enquêteurs ou des brigadiers plus expérimentés de rédiger les rapports de synthèse.

Mais si la polyvalence est indispensable, les membres du groupe reconnaissent également des spécialités individuelles qui s'ajustent en fonction des goûts et des préférences pour certaines tâches. Ce partage des compétences est souvent déterminé par les expériences professionnelles antérieures de chacun. Ainsi, dans un groupe, les gardiens de la paix issus des commissariats ou des Brigades Anti-Criminalité (BAC) seront plutôt assignés aux opérations "musclées » sur le terrain tandis que les officiers se concentreront plus sur les actes procéduraux. Ces spécialisations ou plutôt ces préférences individuelles ne font pas obstacle à la polyvalence. Il s'agit de préférences négociées qui s'expriment dans les discours par la complémentarité.

Chaque groupe d'investigation fixe ses propres règles de fonctionnement. On trouve ainsi des groupes matinaux, débutant leur journée systématiquement ensemble à huit heures tandis que d'autres optent pour une plus grande liberté individuelle, chacun commençant à l'heure qu'il souhaite. De même, un groupe peut traiter collectivement de chaque dossier tandis que dans un autre chaque affaire est distribuée individuellement aux policiers pour être ensuite soumise au collectif, une fois qu'elle est suffisamment avancée. 


\subsection{Les relations entre groupes: la course à la belle affaire, source de motivation et d'émulation entre policiers}

L'évaluation de la structure sur les belles affaires agit sur la motivation des policiers et sur la compétition que cela entraîne entre les groupes et entre les brigades de la sûreté départementale.

La belle affaire étant considérée comme le travail noble dans la profession policière, elle entraîne une forte motivation des policiers. Ceci se manifeste par exemple par la multiplication des heures de travail lorsque lorsqu'ils enquêtent sur des affaires d'envergure. Mais en " dopant » la motivation, la recherche de la belle affaire entraîne également une compétition entre les groupes et les brigades au sein de la structure. Ainsi, lorsqu'un groupe réalise une affaire importante, les autres voudront en faire autant au risque de se voir, à terme, souffrir de la comparaison et donc d'être accusés d'une certaine incompétence.

Il s'opère donc au sein de la structure, une véritable course aux belles affaires qui instaure une émulation entre les groupes qui a des effets opposés sur les résultats de la SD. D'un côté, elle participe aux bons résultats de la structure puisqu'elle pousse les groupes et les brigades à s'aligner les unes par rapport aux autres. Elle maintient donc un niveau de performance constant dans l'organisation. Mais d'un autre côté, la course aux belles affaires peut donner lieu à des conflits qui déstabilisent les groupes et, par conséquent, pénalisent les résultats de la structure. Les deux groupes de la brigade des stupéfiants ont été victimes des affres de la concurrence. Deux jeunes lieutenants furent placés à la tête de leurs groupes suite aux promotions quasi conjointes nomination conjointe des officiers anciens chefs de groupes. Selon les règles administratives c’est le policier le plus gradé 
ou le plus ancien qui doit prendre à la tête du groupe. Les deux chefs de groupe avouent qu'à ce moment, ils se livraient une compétition. Or, un des groupes, le groupe 2 « sort » une affaire très importante de cannabis 2 grosses affaires rapportant au service $11 \mathrm{~kg}$ d'herbe et $120 \mathrm{~kg}$ de cannabis. A ce moment, les acteurs parlent non pas de concurrence mais d'une certaine émulation qui se crée entre les 2 groupes. L'ambiance se dégrade au moment ou le groupe 1 est saisi de deux dossiers importants dont l'un implique une tonne de drogue. Mais le groupe peine dans son dossier. De plus l'enquête est freinée par le fait que par le jeu des mutations se retrouvent avec plusieurs jeunes gardiens de la paix à former. N'avançant pas sur le dossier, ils subissent des railleries de l'autre groupe et cela finit par provoquer un conflit ouvert entre les deux groupes. Le conflit est si important que le commissaire est contraint de nommer un autre capitaine à la tête de la brigade plus dirigiste qui a pour mission de tempérer les ardeurs des jeunes policiers. Cet exemple démontre que la compétition occasionne des conflits qui à son tour dégrade la performance de la brigade.

On s'enlisait dans ce dossier là, on se décourageait, on se remotivait et on se décourageait au final c'est la caractéristique des dossiers très lourds, alors fatigue physique, fatigue nerveuse et psychologique, on s'est engueulé avec l'autre groupe qui nous demandait ce que l'on foutait en nous chambrant jusqu'au jour où on ne pouvait plus. On s'est allumé " Ancien chef de groupe stup 1

La course aux belles affaires entraîne donc un équilibre précaire au sein de la sûreté départementale : elle contribue au maintien un niveau de performance élevé de la structure mais elle peut en même temps la dégrader lorsqu'elle est trop effrénée. 


\section{Les relations extérieures : la sélection des interlocuteurs inter-institutionnels}

\subsection{Les relations avec les commissariats : comment s'alimenter en affaires?}

Les inspecteurs de la sûreté départementale parviennent à trouver les affaires d'envergure grâce aux commissariats locaux. Travaillant principalement sur initiative, les policiers de la sûreté départementale disposent d'une marge de manœuvre importante pour choisir les affaires qu'ils traitent. Les policiers recherchent dans le système d'information interne à la police (les telex) les affaires avec des chefs d'inculpation suffisamment graves pour justifier qu'ils s'en saisissent ${ }^{25}$. Le critère déterminant qui pousse les policiers de la SD a s'intéresser à une affaire est les premiers de l'enquête qui permettent d'évaluer sa faisabilité et son envergure. Pour la BSU, cela peut être des similitudes dans les modes opératoires de braquages de banque répétés. Pour les stupéfiants, cela peut être une saisie de drogue qui laisse envisager des suites importantes. Lorsqu'ils repèrent ce type d'affaire, les policiers de la sûreté contactent, ensuite, les commissariats qui ont communiqués l'information. Les policiers de la SD étant majoritairement issus des commissariats locaux, ils choisissent généralement de contacter leurs anciens collègues pour leur demander des informations complémentaires sur le telex qu’ils ont repéré. ${ }^{26}$.

\footnotetext{
${ }^{25}$ Il peut s'agir par exemple de faits impliquant une pluralité d'auteurs (violences en bandes organisées) ou des infractions répétées (émissions de faux cheques, règlements de compte dans les cités entre bandes rivales) pour la BSU.

${ }^{26}$ A chaque fois l'interlocuteur d'un groupe dans le commissariat sera particulier : par exemple, dans un même commissariat un groupe s'adressera plutôt au GRI (groupe de recherche et d'investigation) tandis qu'un autre s'adressera au SVP (service de voie publique). Le GRI (groupe de recherche et d'investigation) est le service chargé des enquêtes judiciaires. Le SVP (service de voie publique) est le
} 
Récupérer une affaire auprès des commissariats ne va pourtant pas de soi. Faute d'un droit d'évocation qui leur permettrait de se saisir des affaires qu'elles souhaitent, les brigades de la sûreté doivent obtenir l'accord des commissariats. La brigade est donc contrainte de négocier avec les commissariats locaux, afin que ceux-ci les alimentent régulièrement en affaires. 3 éléments rendent possible cette négociation : en premier lieu, pour les commissariats locaux généralement surchargés, confier une affaire à la sûreté est un moindre mal. Ceci est d'autant moins problématique lorsqu'il d'affaires qui requièrent un long investissement en temps et en matériel que les commissariats ne peuvent se permettre. Du reste, la résolution de certaines affaires par la sûreté départementale peut être perçue comme une réelle aide par les policiers des commissariats. Citons ici l'exemple de l'arrestation par le groupe 2 de la BSU de braqueurs très actifs sur lesquels le commissariat ne pouvait enquêter faute de temps et de moyens pour établir des preuves solides

« Vu la complexité de l’affaire, les filoches, les planques nécessaires, on va demander à ce que soit la sûreté qui soit saisie, ça se fait naturellement. Sur certaines affaires on aurait aimé continuer mais on est bien conscient que si on ne peut pas le faire, ils le feront. Par exemple pour l'affaire du " gorille», on aurait repris très difficilement cette affaire parce qu'il y avait 150 jeunes, on en a entendu qu'un et on a eu quelques infos mais pour nous ca nous aurait pris un temps fou. Mais vous savez ici de toute façon on a du stock, on a de la matière alors s’ils veulent en prendre pourquoi pas »») OPJ UVP commissariat.

En second lieu, les relations d'amitié entre les fonctionnaires de la sûreté et des commissariats accélèrent la reprise des affaires.

Mais en troisième lieu, la reprise des affaires s'inscrit dans une relation d'échange plus vaste: pour s'attirer la

service chargé des délits de voie publique 
coopération volontaire des commissariats, les brigades se montrent toujours promptes à les assister sur des affaires lorsqu'ils en font la demande. C'est perçu comme une aide réelle par les commissariats locaux puisque de cette manière la procédure est conclue plus rapidement. Enfin, et surtout, cette collaboration repose surtout sur la réciprocité de l'échange d'informations entre les deux services qui est le fondement du travail policier.

Malgré des points d'achoppement peuvent surgir au moment de la reprise des affaires. En raison de la contrainte du taux d'élucidation, les commissariats peuvent être conduits à conserver une affaire. En effet, nous l'avons vu, le taux d'élucidation est un ratio entre les faits constatés et les faits élucidés ${ }^{27}$, ainsi lorsque la sûreté reprend une affaire au commissariat, celle-ci ne pourra pas être comptabilisée dans ce ratio. Par conséquent, et comme les brigades de la sûreté, les commissariats sont attentifs aux premiers éléments d'enquêtes qui leur permettent d'évaluer si une affaire sera résolu rapidement ou non : des premiers faits vagues ayant peu de possibilités d'être recoupés par des témoignages ou d'autres indices ${ }^{28}$, constituent le plus mauvais cas de figure. À l'inverse, une plainte détaillée avec plusieurs dénonciations offre des points de départ intéressants pour démarrer une investigation. En somme, la rétention d'une affaire par les commissariats dépend du degré d'avancement de l'enquête. Si les premiers éléments de l'enquête conduisent à des pistes exploitables, le service chargé de l'enquête au commissariat aura plutôt tendance à conserver l'affaire malgré les sollicitations de la sûreté départementale. Cette attitude peut amener les deux services à s'opposer puisque les policiers de

${ }^{27}$ Les faits constatés sont les plaintes et les faits élucidés le sont lorsque les personnes reconnaissent les faits ou lorsque la culpabilité des auteurs présumés est établie. Nous expliciterons plus précisément ce mode de calcul dans la partie IV.

${ }^{28}$ Il peut s’agit par exemple, un incendie criminel sans aucun témoin. 
la sûreté départementale sélectionnent aussi leurs affaires en fonction des premiers éléments de l'enquête. Les cas de conflits restent cependant rares. Lorsque ces cas de figure se présentent, les policiers de la sûreté départementale laisseront l'affaire aux commissariats. S'ils se montrent aussi conciliants, c'est essentiellement parce qu'ils craignent de tarir un réseau les approvisionnant en affaire. Du reste, cette attitude est d'autant moins problématique qu'ils ne sont pas eux soumis à une obligation quantitative de résultats.

\subsection{Les relations avec les magistrats ; une affaire de confiance}

Intéressons-nous à présent aux relations des policiers avec les magistrats puisque le code de procédure pénale prévoit une relation hiérarchique entre eux dans la conduite des investigations. Cette relation est régie par une configuration similaire à ce que nous décrivions précédemment à savoir une forte autonomie et un contrôle distant de la part des magistrats ${ }^{29}$

Les magistrats le reconnaissent sans peine, les policiers jouissent d'une autonomie importante sur le terrain. Débordés par leur charge de travail quotidien, les magistrats du TGI ne peuvent exercer un contrôle assidu sur l'ensemble des actes d'investigation des policiers. Les magistrats sont donc dépendants des initiatives des OPJ sur le terrain et de la bonne application des instructions qu'ils leur transmettent. De leur coté, et comme le prévoit le CPP, les policiers sont tributaires

\footnotetext{
${ }^{29}$ Précisons que l'étude empirique sur laquelle nous appuyons nos résultats a été effectuée en 2002, date à laquelle a été introduite des profonds changements dans l'institution judiciaire et en premier lieu, l'instauration des juges de liberté et de la détention (JLD) Loi du 15 juin 2000 - disposition applicable au 1er janvier 2001. Par conséquent, les résultats présentés ici, ne prennent c pas compte du rôle du JLD dans les relations policiers-magistrats.
} 
de l'accord des magistrats pour toute une série d'actes : faire des perquisitions, obtenir les prolongations de gardes à vue (GAV) qu'ils requièrent, ou encore - dans le cadre des Commissions Rogatoires (CR)- faire des écoutes lorsqu'ils les jugent utiles. Autrement dit, le déroulement de l'enquête policière dépend en grande partie des décisions du magistrat, puisque, possédant la légitimité juridique, il aura toujours le dernier mot en cas de litige.

Du fait de cette étroite interdépendance, coopérer harmonieusement devient une nécessité pour les deux acteurs. Sur ce point, les policiers jugent très différemment les différents magistrats du TGI et ils revendiquent leurs préférences pour leur directeur d'enquête. C'est pourquoi, les policiers des deux brigades travaillent, la plupart du temps, avec les mêmes magistrats, ceux avec lesquels ils entretiennent des relations de confiance. Il se forme alors des binômes de collaboration qui se forment par un processus de sélection réciproque entre policiers et magistrats.

Les policiers de la sûreté départementale parviennent à choisir leur directeur d'enquête grâce au système des permanences du substitut du procureur : les permanences des magistrats d'astreinte sont notifiées sur un tableau affiché dans l'ensemble des services de police de la circonscription. Ceci leur permet de faire ouvrir une information judiciaire avec substitut ou un juge d'instruction particulier. Concernant ces derniers, le CPP prévoit que le doyen des juges d'instruction désigne le juge qui sera assigné à une affaire. Pourtant, dans la pratique, les juges d'instructions sont aussi soumis à un système de permanence. Par conséquent, pour travailler avec un juge en particulier, il suffit de demander au procureur l'ouverture de l'information judiciaire la semaine où le juge souhaité tient sa permanence. La sélection du directeur d'enquête est un enjeu important dans les dossiers importants. 
«Y'a des magistrats qu'on sent moins bien, c'est une question de personne, il sera par exemple plus ou moins ouvert, il y en a qui sont noyés sous les dossiers et les relations humaines ils s'en foutent, mais c'est rare et ca s'estompe parce que sur une grosse affaire tu les as toujours au téléphone. Au parquet de Versailles c'est rare. Alors on regarde qui est de permanence, en fonction de la nature du dossier et ce qu'on veut en faire » Chef de groupe BSU 1

Chaque brigade n'a pas la même liberté de choisir « son » magistrat. Travaillant surtout sur initiative, la BSU a plus de loisir de choisir son directeur d'enquête. À l'inverse, la brigade des stupéfiants dispose de moins de liberté puisqu'elle est saisie beaucoup plus par des commissions rogatoires comme nous l'avons vu précédemment. Pour les policiers cela peut être une contrainte puisqu'ils doivent s'adapter aux exigences professionnelles à chaque fois spécifiques des juges d'instruction. Ce n'est que lorsqu'elle travaille d'initiative que la brigade peut choisir le juge avec lequel elle souhaite travailler.

De leur coté, les magistrats sélectionnent aussi le service de police d'abord en fonction de sa spécialité : les affaires qui requièrent des investigations plus longues sont confiées aux services de police judiciaire, comme la sûreté départementale ou la police Judiciaire alors que les commissariats sont (dans la grande majorité des cas) cantonnés aux "petites affaires". Les magistrats ont également la possibilité d'affiner ce choix en adressant leurs saisines à un service de police particulier voire à une unité particulière qui a leur préférence. À la sûreté départementale, un magistrat peut ainsi choisir le groupe particulier d'une brigade avec lequel il souhaite travailler. Ici aussi cette sélection n'est pas systématique. Elle s'applique pour les dossiers qui suscitent un intérêt particulier pour le magistrat.

Si ces deux acteurs attachent autant d'importance à choisir leur partenaire d'enquête, c'est en raison de ses 
aptitudes professionnelles qui ont été préalablement évaluées et éprouvées. Comme nous le précisions précédemment, les magistrats ne peuvent pas contrôler de manière "rigide " chaque acte des policiers et ceux-ci ont besoin d'une forte autonomie dans leurs enquêtes. Dès lors, nouer une relation de confiance devient la règle d'or entre les deux parties. Dans cette relation, la confiance n'est pas un attribut. Elle se construit autour d'un certain nombre d'exigences mutuelles apprises dans la gestion quotidienne des dossiers. Plaçonsnous du côté des deux acteurs pour comprendre quelles sont ses exigences vis-à-vis de son partenaire.

L'exigence première des juges d'instruction vis-à-vis des policiers tient à la qualité de la procédure qu'ils leur remettent. Selon le jargon policier, la procédure doit alors être «carrée ». Cela implique d'une part, qu'elle ne doit pas comporter de vices de procédures pouvant conduire à l'annulation d'un acte. D'autre part, que «toutes les portes doivent être fermées », c'est-à-dire qu'à la lecture du document, toutes les hypothèses relatives à l'enquête aient été envisagées, vérifiées et recoupées. De plus, les éléments avancés dans le dossier (témoignages, saisies..) ne doivent laisser aucun doute sur la charge de la preuve. Mais pour les magistrats du parquet, "faire confiance » aux policiers doit également se comprendre comme un choix par défaut: débordés par le flux harassant de dossiers quotidien, les magistrats n'ont également pas d'autres choix que de laisser une autonomie forte aux policiers dans la conduite de leurs investigations.

De leur coté, les policiers attendent des magistrats qu'ils prennent en compte leurs avis dans les affaires en cours. Au moment de notre étude et avant l'apparition des juges d'application des peines, ceci était particulièrement frappant pour les décisions de remise en liberté du mis en cause. Par exemple, lors de l'interrogatoire de première comparution qui 
clôt l'instruction préparatoire, les policiers peuvent souhaiter l'emprisonnement du mis en cause grâce au mandat de dépôt, s'ils estiment, le cas est fréquent dans les affaires de stupéfiants, qu'il peut prévenir ses complices. Inversement, et toujours dans le même type d'affaire, les policiers peuvent solliciter la clémence du juge ou d'un substitut à l'encontre d'un informateur ${ }^{30}$. Autre exemple, les policiers doivent solliciter l'autorisation du juge pour perquisitionner. Or, et en fonction des circonstances, ils n'ont toujours pas systématiquement le temps de solliciter l'avis du juge. Aussi, lorsqu'ils ont la confiance d'un juge, ils prendront l'initiative seuls de perquisitionner. Dans le cas contraire, ils se raviseront en prenant le risque de compromettre l'enquête. D’une manière plus générale, pour obtenir la confiance des policiers, les magistrats doivent donc de prendre en compte leurs avis dans la conduite de la procédure judiciaire.

«On écoute les OPJ lorsqu’on arrive lors des déferrements, après GAV. L'OPJ a eu pendant 24 heures, 48 parfois 4 jours en matière de stups l'intéressé avec lui. Donc il l'a entendu à plusieurs reprises, donc il a pu cerner la personnalité de l'intéressé, donc, moi, j'aime bien avant de recevoir les gens faire un point avec les enquêteurs, sur l'enquête et sur les faits certes, mais aussi sur la personnalité : "Comment vous le ressentez? " "Il est bien ou au contraire il cache des choses ", « il essaye de protéger machin ou il est franc » " c’est un type intelligent ou il ne répond jamais aux questions »... C’est un échange de points de vue, mais ça ne va pas plus loin, je ne fais pas ma religion uniquement au vu de la relation que peut me faire l'OPJ. » Juge d'instruction

«Si t’as bien travaillé les gens iront au trou sinon ils n’iront pas. Par exemple il y a un juge que l'on déteste. Pourquoi ? Parce qu'elle (le juge) nous a foutu une nana dehors alors qu'elle avait tiré une balle dans la tête d'un autre mec. On avait un tuyau sûr. On savait que c'était elle parce qu'elle était

${ }^{30}$ Ce cas de figure est très fréquent chez les stupéfiants où l'informateur occupe une place centrale dans les investigations 
amoureuse du mec qui l'avait trompée. Elle (le juge) a dit : « Elle est jeune et elle ne savait pas ce qu'elle faisait. » Alors elle l'a remise dehors. Bon elle nous avait déjà fait le coup... Une fois ça va mais deux fois comme elle nous l'a fait, faut pas pousser. » OPJ BSU

La confiance ne s'acquiert que par apprentissage successif entre les acteurs. Elle sera mise progressivement à l'épreuve lors des premières collaborations où ils se testeront mutuellement pour vérifier si leurs attentes successives sont respectées. La confiance ne s'acquiert pas seulement grâce au respect des exigences mutuelles professionnelles réciproques. La subjectivité et l'apprentissage entre également en compte dans cette relation. La comptabilité des caractéristiques personnelles (l'humour, les goûts) renforce la relation de confiance.

Malgré cette confiance, il s'agit bien d'un rapport de subordination. D'une part, l'expertise des magistrats qui leur sont supérieurs en matière juridique qui fait d'eux les « véritables » directeurs d'enquête comme le reconnaissent les policiers. Mais d'autre part, on pourrait penser que l'autonomie élargie des policiers puisse conduire à certaines dérives comme des «bavures" ou des entorses aux procédures juridiques récurrentes par exemple. Or, dans les faits, même si celles-ci existent nous avons pu l'observer, elles ne sont ni suffisamment nombreuses ni flagrantes pour qu'elles fassent l'objet de sanctions exemplaires. Comme l'expliquent les policiers, ceci s'explique par leur souhait de conserver la confiance des magistrats : s'ils commentaient en effet, des violations trop flagrantes, ils pour conserver la confiance avec les magistrats comme celle de leur hiérarchie interne, les policiers se doivent de rendre des procédures « carrées », respectant à la lettre les règles du CPP. 


\section{Questions}

1. Comment expliquez-vous l'autonomie élagie des policiers au sein de cette structure ? Comment s'exerce le contrôle du travail si la hiérarchie accorde autant d'autonomie aux policiers?

2. Sur quels éléments repose l'efficacité des policiers de cette structure ? Parmi ceux-ci, quel serait l'élément " clé »?

3. Quelles sont les limites que vous voyez au maintien de la performance dans cette structure?

1. Dans cette bureaucratie policière, les agents disposent d'une large autonomie et que leurs hiérarchies - aussi bien le commissaire de la sûreté départementale que les magistrats du parquet optent pour un contrôle distancié. Cette configuration particulière d'une autonomie élargie et d'un contrôle hiérarchique s'explique avant tout par la nature du travail d'investigation. En effet, le travail policier reposant essentiellement sur l'initiative des policiers, la hiérarchie de la structure ne peut prescrire l'ensemble des tâches à accomplir et n'a pas d'autre choix que d'accorder une forte autonomie aux fonctionnaires.

Mais la distance du contrôle hiérarchique ne disparaît pas mais il s'exerce sous la forme d'accords tacites que l'on peut regrouper en 2 catégories : un contrôle par les résultats, un contrôle par la confiance.

Premièrement l'autonomie est accordée par la hiérarchie moyennant l'obtention de résultats en termes de «belles affaires ». Or, nous avons établi que résoudre des belles affaires constituait une motivation particulière pour les policiers puisqu'il s'agissait d'une forme d'intérêt intrinsèque à la profession. Particulièrement motivés par cette tâche, les 
« contrôlés » n’ont plus réellement besoin de l'être sur ce point et ce d'autant moins qu'elle entraine une émulation entre les groupes qui permet de maintenir un niveau de performance continue dans les brigades. Autrement dit, et du fait du mode d'évaluation tout à fait particulier (une évaluation réputationnelle), il s'opère plus précisément une convergence d'intérêt entre les policiers et leurs hiérarchies qui donne lieu à cette configuration de relation hiérarchique particulière : autonomie large et contrôle distant. Par conséquent, dans cette structure, subordonnés et hiérarchies convergent vers un but commun : résoudre de belles affaires.

Deuxièmement et dans le cas de la relation plus particulière entre policiers et magistrats, c'est la confiance fait office de contrôle dans la conduite des enquêtes : pour les policiers, la confiance que leur accorde les juges, leur permet de disposer d'une grande autonomie dans leurs enquêtes qui concoure directement à leur efficacité puisque cela leur permet d'employer (en grande partie) les moyens qu'ils jugeront utiles pour mener à bien leurs enquêtes (écoutes, garde à vue etc.). Aussi, si les policiers venaient à violer les règles juridiques qui encadrent le $\mathrm{CPP}$, ils prennent le risque de rompre la confiance des magistrats, ce qui aurait des conséquences désastrueuses sur leurs performances puisque non seulement ils prendraient le risque que les magistrats ne leur confieraient plus d'enquêtes d'envergure (susceptible de devenir une belle affaire) ou qu'ils restreignent leurs libertés d'action dans leurs enquêtes. Par conséquent, pour conserver la confiance des magistrats, les policiers doivent respecter la règlementation $^{31}$ (rendre des procédures "carrées "

\footnotetext{
${ }^{31}$ Ce point mériterait à notre sens un approfondissement concernant les effets de cette relation de confiance dans le traitement des procédures ? Nous manquons de données pour approfondir ce point mais nous émettons les hypothèses suivantes : il s'agit tout d'abord des «entorses » à la législation puisque les magistrats -devant faire preuve d'empathie vis à vis du travail policier- peuvent fermer les yeux sur des irrégularités exceptionnelles de policiers qu'ils jugent compétents et qu'ils ne
} 
notamment), ce qui retire à la hiérarchie une possibilité de contrôle.

En somme, les fonctions traditionnelles du contrôle hiérarchique que sont l'obtention de résultats, le respect des règles, l'investissement au travail se déplacent au sein des règles qui régissent l'autonomie des policiers. Autrement dit, la régulation autonome (Reynaud 1997) s'autocontrôle ellemême en grande partie : les « contrôlés » n’ont plus besoin de l'être ou, en tout cas, pas dans les limites généralement imposées par la bureaucratie. . Ceci se rapproche de la notion de contrôle latéral développé par Lazega qui met en exergue les systèmes de surveillances et de sanctions qui s'établissent entre pairs (Lazega 1999) (Lazega 1999). Mais ce déplacement n'est possible que par la compétence éprouvée par la hiérarchie des policiers de cette structure. On peut par conséquent considérer que c'est la compétence qui est au cœur de l'accord implicite qui instaure un contrôle distant. Comme le fait remarquer De Terssac, la compétence sert ici de «monnaie d'échange entre l'encadrement et les exécutants » (De Terssac 1992 P. 185).

2. Cette organisation parvient à être performante en raison d'un système d'évaluation basé sur la qualité des affaires qui permet à un intérêt intrinsèque (le beau cas, la belle affaire) à s'exprimer. Cela donne lieu à un système d'action concret vertueux pour la performance qui peut se

souhaitent pas trop sanctionner. Pour le fonctionnement du TGI, d'autres conséquences découlent de cette relation mais les données empiriques que nous avons recueillies pour cet article, ne nous permettent pas d'aller plus loin. Nous pouvons cependant avancer l'hypothèse que les magistrats «favoris » des policiers (spécialisés) doivent faire face à un problème de surcharge de travail. Enfin, une autre question découle de cette hypothèse : le processus de sélection réciproque peut-il entraîner un certain cloisonnement entre les magistrats «favoris » des policiers et leurs collègues ne bénéficiant pas des mêmes égards ? 
comprendre comme la combinaison de 3 éléments étroitement liés les uns aux autres :

Premièrement, le mode d'évaluation de la structure construit (en grande partie par défaut) sur leur réputation à résoudre des affaires d'envergure, les «belles affaires", stimule la motivation des policiers puisqu'elles correspondent à un intérêt professionnel intrinsèque de la profession que nous avons définit comme le chef d'œuvre au sens premier du terme.

Ceci donne lieu, deuxièmement, à une émulation ou une compétition et entre fonctionnaires qui renforce un peu plus leur investissement au travail. La course aux belles affaires entraîne, alors deux type d'effets vertueux pour la performance des brigades de la SD. Tout d'abord, elle permet de maintenir un certain niveau de performance dans les brigades puisque les groupes les moins performants auront tendance à s'aligner sur les meilleurs. Ensuite, la compétition que se livrent les brigades et les groupes éloigne la hiérarchie de sa fonction de contrôle traditionnelle (résultats, motivations, règlementation), ce qui leur confère une large autonomie mise à profit pour choisir leurs interlocuteurs inter institutionnels, les commissariats et les magistrats, ce qui est un rouage essentiel de leur performance. En effet, et d'une part, les policiers récupèrent auprès des commissariats des affaires qui les intéressent, celles qui sont susceptibles de devenir une "belle » affaire ». D'autre part, en nouant des relations de confiance avec les magistrats, ils jouissent d'une plus grande liberté d'initiative dans leurs enquêtes qui peut favoriser leur résolution.

Troisièmement, le fait que la structure traite essentiellement d'affaires d'envergure lui donne une visibilité médiatique qui accroît le nombre de postulants potentiels. Outre les opportunités de mobilité professionnelle que représente un poste dans cette structure, cette publicisation 
renforce le sentiment des candidats qu'ils pourront exercer la partie noble du métier de policier. Par conséquent, les postulants étant nombreux, les chances de recruter des policiers compétents sont plus importantes. Le recrutement par cooptation appliqué par les brigades, leur apportent certaines garanties sur la capacité du candidat à s’intégrer dans le groupe. Ceci garantie donc une construction harmonieuse des règles collective qui régissent leur autonomie, une condition nécessaire dans la réussite des brigades.

3. L'agencement vertueux des différents éléments du système (cooptation, sélection des affaires, concurrence) n'est pas mécanique. Il est plutôt instable et doit se comprendre comme un équilibre dynamique précaire pouvant être déstabilisé par les mêmes éléments qui le créait et en premier lieu desquels se place la concurrence. En effet, la compétition qui s'instaure dans la course aux belles affaires lorsqu'elle est trop poussée se transforment en conflits qui peuvent remettre en question l'équilibre des accords de groupes ce qui, consécutivement, pénalise leurs résultats et plus généralement l'ensemble de l'équilibre vertueux que nous décrivions. Nous avons cité le cas des groupes de la brigade de stupéfiants qui, suite à une série de conflits trop importants provoqués par une compétition trop effrénée, avaient été profondément renouvelés en hommes. En somme, si la compétition est source de performance, elle contient ses propres limites capables de briser la mécanique systémique des effets vertueux. 


\section{Bibliographie}

BRODEUR J.-P., 1984, La police : mythes et réalités, Criminologie, XVII, 1.

DUBARD C., TRIPIER P. 1998 «Sociologie des professions », Paris, A. Collin.

JANKOWSKI B., 1996, «Les inspecteurs de police : contraintes organisationnelles et identité professionnelle », Déviance et Société, N²0/1, P. 17-35

JOBERT, B., LECA J., "Le dépérissement de l'Etat à propos de "L'acteur et le système" de Michel Crozier et Erhard Friedberg", Revue Française de Science Politique, Vol. 30, n 6, décembre 1980, p. 1125-1171

LAZEGA. E, 1999 : "Le phénomène collégial : une théorie structurale de l'action collective entre pairs" ; Revue Française de Sociologie, XL-4.

LEVY, R, 1987, «Du suspect au coupable : le travail de la police judiciaire », Paris, Déviance et société Editions Médecine et hygiène.

MINTZBERG H., 1982, «Structure et dynamique des organisations ", Paris, les éditions d'organisations.

MONJARDET D., 1996, " Ce que fait la police : sociologie de la force publique », Paris, La Découverte.

MONJARDET, D., 1988, « Professionnalisme et médiation de l'action policière ", Les cahier de la sécurité intérieure, P. 2149.

MOUHANNA, C, 2001 «Police judiciaire et magistrats : une affaire de confiance ", Mission de recherche "droit et justice », Paris, La documentation Française. 
MOUHANNA C. ; MATELY J.H, 2007 «Police : des chiffres et des doutes ", Paris, Michallon.

MUCCHIELLI, L, 2006, « l'élucidation des homicides : de l'enchantement technologique à l'analyse des enquêteurs de police judiciaire », Déviance et Société, Vol 30, N 1, p 91-119.

REYNAUD J.D., 1997, «Les règles du jeu », Paris, Armand Collin.

TERSSAC De G., 1992, «L'autonomie dans le travail », Paris, PUF. 